\title{
Eastern Cape treatment dysfunction boosts virulent new XDR-TB strain
}

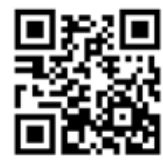

At least half of all known drug-resistant tuberculosis (DR-TB) patients in the Port Elizabeth area (and possibly the entire Eastern Cape) are being treated with too few drugs, fuelling the spread of extensively drug-resistant (XDR)-TB and condemning them to death.

Similar dynamics, though not quite as alarming, pertain in KwaZulu-Natal and to a lesser extent in the Western Cape. An atypical (Beijing) strain of multiple-drug-resistant (MDR)-TB, first detected by researchers at Stellenbosch University 2 years ago, is being created via a 'one-size-fits-all' system of decentralised, nurse-led TB clinics with an insufficient array of drugs and wholly inadequate expertise. In the Eastern Cape, the situation is being worsened by a woeful lack of local political will and/or understanding resulting in a virulent, 'export-ready' budgetcrippling potential domestic time bomb. Researchers spoken to by Izindaba say that patients carrying this 'aberrant' pre-XDR MDR strain and/or the ensuing full-blown XDR are being detected in significant numbers in the Western Cape metropole, the primary destination for the Eastern Cape's huge migrant worker population. The Beijing strain is one mutation away from XDR and is being driven by treatment with an insufficient array of drugs. Izindaba has reliably learnt that the National Health Laboratory Service (NHLS) in Port Elizabeth conducted a Line Probe Assay (using the latest Hain LifeSciences technology) on two locally predominant MDR-TB strains, one in particular (Beijing) described as 'pre-programmed to become XDR'. The conclusion of their lab technicians? The Port Elizabeth (PE) metropole, in particular, has a 'real problem'

\section{In the Eastern Cape, the situation is being worsened by a woeful lack of local political will and/ or understanding - resulting in a virulent, 'export-ready' budget- crippling potential domestic time bomb.}

\section{When slower is better}

'It appears to be fuelled by our patients being put onto a standard regimen of treatment in which very few drugs are effective, one source said. An infectious diseases doctor working in the Eastern Cape explained that nurses could wait for up to 6 weeks for culture results to confirm the DR strain. This 'archaic' confirmation followed the 2-hours-or-less GeneXpert result confirming rifampicin resistance. While the samples were at the laboratory to confirm or identify additional resistance markers by culture, nurses and doctors were 'blindly' putting patients onto standard MDR-TB treatment - inadequate to treat many of the circulating strains and thus amplifying resistance. Médecins Sans Frontières (MSF) is strengthening its MDR drug regimen in the Western Cape, leading the way in combating this new pre-XDR strain. (National policy on TB treatment is to get as many patients onto treatment as soon as possible and to refer patients, but some experts believe there is insufficient emphasis on, and education about, the multiple complexities of drug resistance.) The national guidelines encourage NGOs and provincial clinics to promote nurse-initiated DR-TB treatment based on single GeneXpert results. However, most healthcare workers, including many doctors, do not understand how to tailor MDR treatment based on mutational analysis, resulting (in the case of the Beijing strain) in the rough equivalent of pouring petrol onto a veld fire, the Izindaba sources claim.

\section{First doing more harm ...}

One as yet unpublished research paper on the aberrant MDR-TB strain shows that 25\% of the PE laboratory samples examined are pre-XDR and $25 \%$ are full-blown XDR clearly demonstrating that half of all the cases scrutinised are being inadequately/ incorrectly treated.

A doctor source at the coalface explained that 'essentially treatment has selected this strain out - the issue here is the current system not dealing with the current problem. He said that infrastructure issues, misguided provincial budget allocation, insufficient training and staff, and a general lack of MDR treatment knowledge on the part of both nurses and doctors aggravated matters. The latest available data from the Jose Pearson TB Hospital in PE show that in 2011, just 35\% of MDR TB patients who completed 24 months of treatment remained cured after a year, while only $12 \%$ of XDR-TB patients who completed 24 months of treatment remained cured a year later. In 2012, this treatment 'success' for MDR patients dropped to $29 \%$ and for XDR patients to $9 \%$. This is roughly in line with much of the published data. (The implication is that the remainder have died, will die or have been lost to follow-up, unless the debilitated survivors can quickly access the latest drugs now coming on line bedaquiline and linezolid.)

According to the treatment register at the Jose Pearson TB Hospital, there were 24 confirmed cases of XDR-TB (of which 17 were HIV-positive) in the last quarter of last year. The number of patients started on treatment in the first quarter of 2014 was 48. The hospital sees up to 15 new cases of XDRTB per month, draining a huge area in the western part of the province - from Graaff Reinet to Port Alfred and from Humansdorp to Kareedouw. The clinicians emphasised that this probably represents the tip of the iceberg' in terms of actual MDR/XDR-TB prevalence. Many TB sufferers never make it to a clinic or present too late, having ignored the initial night sweats or the persistent cough. 'TB is a classic for presenting too late. Active case finding needs to be drastically addressed, says Prof. Rob Warren, associate professor in the Department of Medical Biochemistry at Stellenbosch University, whose team first identified the aberrant preXDR-TB Beijing strain at the heart of the latest development.

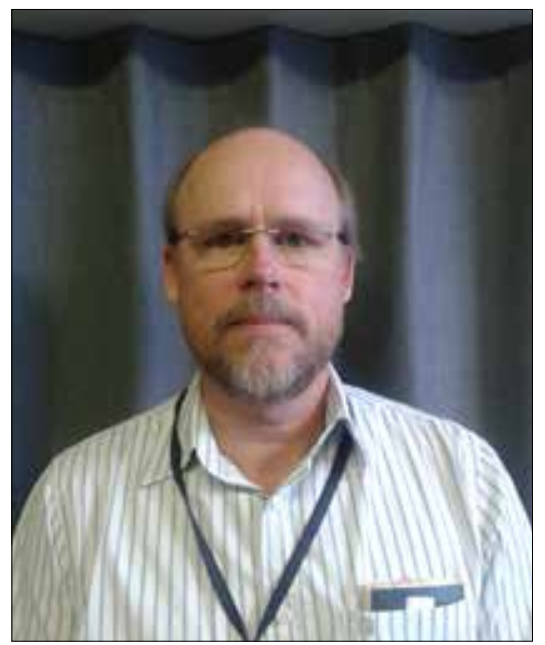

Prof. Rob Warren, associate professor in the Department of Medical Biochemistry at Stellenbosch University. 


\section{'A tide ... when taken at the flood ...?}

The Eastern Cape coalface doctor warned: 'We're at a crossroads. If we don't have a complete revamp of how we approach this problem, we'll lose a golden opportunity to really make a difference. We have a threshold; something needs to be done now. If we continue blindly on, we'll expand resistance to the new drugs we have ... Chief among the solutions, he believes, is a centralised system to allow the appropriate use of new drugs in a regulated manner based on better diagnostics with good monitoring and follow-up (at least until better training and systems are in place to allow more widespread use of the new drug resources). He said patients needed to be put on the correct therapy as soon as possible, with much-improved case-finding and follow-up.

\section{Eastern Cape a TB treatment 'black hole'}

One doctor typified the Eastern Cape (when compared with the other high-TB-burden provinces of the Western Cape and KwaZuluNatal) as 'a black hole - it's left up to poorly trained people to implement a very difficult programme. The historical/political context is showing. The other two provinces have 'a lot of academic research going on and use their resources more efficiently. In the Eastern Cape programmes are poorly applied and badly implemented - to the point where they don't work. My sense is that things are getting worse.' Eastern Cape doctors said that staff shortages and a lack of ongoing monitoring have rendered contact tracing and follow-up wholly ineffective. Warren's team found that the Beijing MDR/XDR-TB strain was causing a huge amount of primary transmission, not only within a specific HIV-positive cohort of patients, but across the board. His team's research centres on novel strategies to reduce the time-to-detection period of both drugsensitive and DR-TB - and to understand the causative agents of the disease better. Their goal is to 'develop a foolproof diagnostic method that can be used by anyone, anywhere'.

\section{TB diagnosis 'behind the research curve'}

The current TB diagnostic method remains an arduous process that requires the patient to present to the health facility for screening, diagnosis and treatment. Sputum samples are sent to a peripheral laboratory for GeneXpert testing and to a central laboratory for drug susceptibility testing. The patient is therefore not present when the results arrive. Up to 20\% of patients do not return at all for their results, and even top government TB Directorate

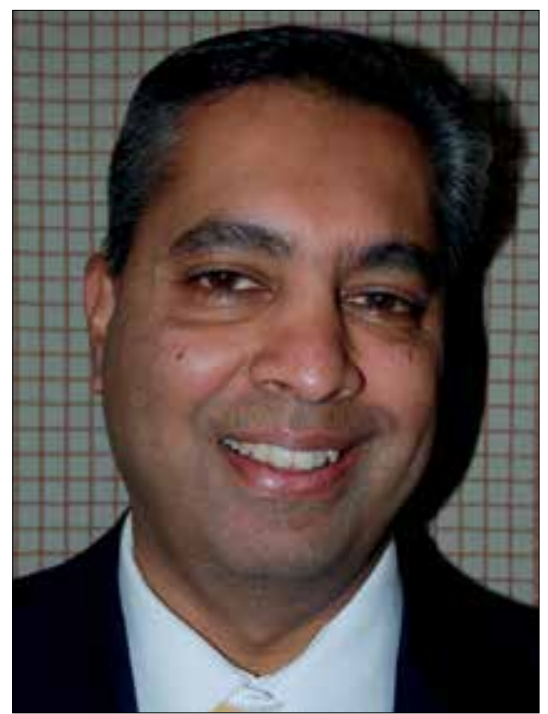

Prof. Keertan Dheda, Head of Pulmonology, University of Cape Town and Groote Schuur Hospital.

officials admit that the systems for tracing them are weak. On testing, Warren observed: 'The tools are out there. There's the first-line probe and then the probe for second-line drugs - if we implemented the second-line test, we could probably reduce the [laboratory test] turn-around time from around 50 days to 3 days.' The test is not perfect yet (a reason given for its low use in spite of its being approved by the World Health Organization (WHO)), but it picks up over $80 \%$ of secondline resistance (both are Hain LifeSciences assays). Warren emphasised that in the South African (SA) TB 'outbreak' context, the success rate would probably be much higher because outbreaks generally contain the same mutations, making identification much easier.

\section{Assuming proper adherence to} national DR-TB management guidelines, the current per patient cost of XDR-TB is ZAR303 508 (USD26 392), four times greater than MDR-TB at ZAR77 878

(USD6 772), and 103 times greater than drug-sensitive TB at ZAR2 955 (USD257).

An Izindaba canvassing of some top TB researchers revealed that they do not necessarily have 'the ear' of the National Department of Health (NDoH). Said Warren: 'Unfortunately there isn't good liaison between research, and policy and practice. Things filter slowly through the academic journals into their expert group discussions where they may or may not take on an idea and put it forward.'

\section{XDR-TB on the increase nationally}

Between 2007 and 2012, SA's recorded cases of MDR-TB (i.e. resistant to at least two of the primary drugs used to combat standard TB) almost doubled. SA has the highest incidence of TB among the 22 high-burden countries $(860 / 100000)$, and when adjusted for population, it outdoes India and China. According to 2012 government figures, just $42 \%$ of patients diagnosed with MDR-TB began treatment. Observes Dr Gilles van Cutsem, MSF's medical co-ordinator for SA and Lesotho: 'We have in SA one of the only rising epidemics of drug-sensitive $\mathrm{TB}$ and DR-TB. And we're not doing very well at detecting it and treating it.'

In a highly cited paper $^{[1]}$ co-authored by Prof. Keertan Dheda, Head of the Lung Infection and Immunity Unit in the University of Cape Town's Lung Institute, the budgetary implications of not responding appropriately to the DR-TB challenge are daunting. Assuming proper adherence to national DR-TB management guidelines, the current per patient cost of XDR-TB is ZAR303 508 (USD26 392), four times greater than that of MDR-TB at ZAR77 878 (USD6 772), and 103 times greater than drug-sensitive TB at ZAR2 955 (USD257). Despite DR-TB's comprising only $2.2 \%$ of the case burden, it consumed $32 \%$ of the total estimated 2011 national TB budget of ZAR2.5 billion (USD218 million). A frightening $45 \%$ and $25 \%$ of the DR-TB costs were attributed to anti-TB drugs and hospitalisation, respectively. XDR-TB consumed $28 \%$ of the total DR-TB diagnosis and treatment costs. Laboratory testing and anti-TB drugs comprised the majority (71\%) of MDR-TB costs, while hospitalisation and anti-TB drug costs comprised the majority (92\%) of XDR-TB costs. A decentralised XDRTB treatment programme could potentially reduce costs by ZAR79 695 (USD6 930) (26\%) per case and reduce the total amount spent on DR-TB by $7 \%$, the authors conclude. ${ }^{[1]}$

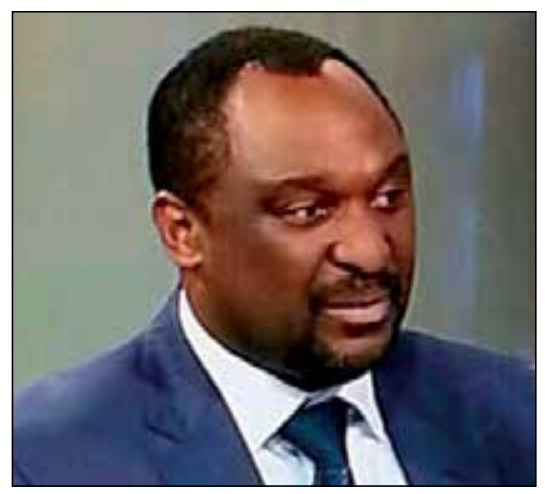

Dr Norbert Ndjeka, head of the National MDRTB Directorate. 


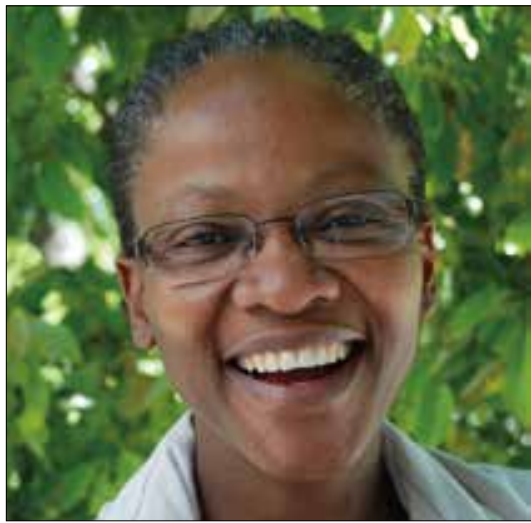

Dr Lindiwe Mvusi, National TB Directorate chief.

\section{SA's TB control 'a} comedy of errors'

Both Warren and Dheda have emphasised that unless the drug-susceptible 'TB tap' is turned off, DR-TB will continue to grow. Dheda's work currently centres on creating an effective new combination regimen for DR-TB.

In a paper published in the South African Respiratory Journal, ${ }^{[2]}$ Warren and his coauthors bluntly say that their review of $\mathrm{TB}$ statistics 'suggests that the current National Tuberculosis Control Programme is unable to curb the emergence and spread of this difficult-to-treat epidemic'. The country's TB control history was 'a comedy of errors', which more modern technology could have helped avoid. 'It is therefore essential that we learn from these mistakes and use current knowledge to design diagnostic algorithms and treatment guidelines that prevent acquisition of resistance and ensure improved treatment outcomes', they conclude. ${ }^{[2]}$ Warren emphasised that he was not blaming anyone in the current national TB Directorate, but merely pointing to the treatment mistakes of the past that they were now saddled with.

Neither Dr Norbert Ndjeka, head of the MDR-TB Directorate, nor Dr Lindiwe Mvusi, National TB Directorate chief, had seen the PE NHLS research paper - but they were eager to study it. Ndjeka said the national guidelines 'clearly stated' that the standardised regimen was for newly diagnosed MDRTB patients only. MDR-TB patients exposed to second-line drugs, pre-XDR-TB and XDR-TB were treated with an 'individualised regimen' (however, Izindaba established that this was only after drug susceptibility results were made available after a wait that could take 6 weeks - again, it is in this time that patients are being 'undertreated'). Ndjeka denied that many clinicians lacked sufficient knowledge, describing them as 'well trained, and educated'. He said he was due to hold a workshop with his provincial staff and clinicians in Johannesburg in midto late February - and invited Izindaba to bring Warren and its other sources to address them. He described the Jose Pearson Hospital MDR/XDR figures cited as 'another gross inaccuracy', saying last year's WHO Global Tuberculosis Report had SA's 2011 cohort analysis and that the 2012 report was still incomplete, but due for finalisation this April.

Ndjeka said that SA was a 'very high' MDR-TB country, and his directorate had consulted the 'best brains in the country' on this issue. 'We cannot work with each and everybody, though the top ones are in constant communication with us - I can provide proof of this', he asserted. He said that describing the country's TB control history as a 'comedy of errors' was 'malicious'. 'We need experts who provide solutions, given the little existing evidence,' he added. Mvusi conceded that the Hain Line Probe Assay, version 2, would 'help greatly in reducing the waiting time for confirmatory first- and second-line drug susceptibility testing because the patient could then be switched to the appropriate regimen earlier'. Ndjeka said that linezolid and bedaquiline were being introduced 'in a very responsible manner' in the public sector, SA being the first TB programme in the world to introduce new agents in about 40 years.

\section{In the Eastern Cape, the situation is being worsened by a woeful lack of local political will and/ or understanding - resulting in a virulent, 'export-ready' budget- crippling potential domestic time bomb.}

\section{Eastern Cape admits there's a problem - but it's 'being addressed'}

An Eastern Cape health department spokesman revealed that bedaquiline had already been 'responsibly' introduced at the Jose Pearson Hospital (and a few other selected hospitals nationwide), and 'so far no adverse events have been reported'. TB clinicians across the country had just met to discuss the scale-up of linezolid and bedaquiline. The Jose Pearson Hospital 'performance figures' quoted by Izindaba were 'acknowledged', but the data were 'currently being cleaned up to April this year before being submitted as final to the WHO'. He said that the province had a very high MDR-TB burden and admitted that treatment outcomes were poor - but a 'lot of progress' in managing the disease had been made in recent years. The NHLS had produced evidence that positive GeneXpert rifampicin-resistant cases were more likely to be MDR-TB, 'hence our guidelines recommend starting MDR-TB treatment, taking a sample for confirmatory test through the Line Probe Assay or conventional drug susceptibility testing. He repeated the $\mathrm{NDoH}$ assertion that MDR-TB patients exposed to second-line TB drugs, pre-XDR TB patients and XDR-TB patients were treated with an individualised drug regimen. An expert provincial MDR-TB committee met every quarter to review and discuss complicated cases and facilitate/regulate access to the new drugs. The reported increase in DR-TB in the province between 2007 and 2012 was actually an indication of intensified case finding and better diagnostic technology. Active steps to address the challenges included partnering with the NHLS to improve the response to positive DR-TB results with rapid follow-up diagnostics. The DR-TB decentralisation programme involved ten hospitals, with intensive training of healthcare workers on MDR-TB management being accelerated, while outreach vehicles had been hired to address late presentation of patients and improve case finding and contact tracing. Four hospitals were either under construction or renovation to comply with national infection prevention and control guidelines.

Although Izindaba did not source its most alarming data directly from the PE laboratory, technicians there received a stern warning: 'Permission is required to conduct research or make use of departmental data/ information - this being granted only on condition that these findings are shared with provincial health management before being presented on any platform.'

Izindaba has chosen to withhold the names of some of its Eastern Cape sources, who said they fear becoming targets of angered local officialdom - historically a common anxiety among that province's many deeply committed healthcare workers.

\section{Chris Bateman}

chrisb@hmpg.co.za

S Afr Med J 2015;105(3):165-167. DOI:10.7196/SAMJ.9475 1. Pooran A, Pieterson E, Davids M, Theron G, Dheda K. What
is the cost of diagnosis and management of drug resistant
tuberculosis in South Africa? PLoS One 2013;8(1):e54587.
[http://dx.doi.org/10.1371/journal.pone.0054587]
2. Warren RM, Hoek K, Sirgel F, et al. Emergence and treatment of
drug resistant tuberculosis: A comedy of errors. South African Respiratory Journal 2011;16(4):112-116. 